\title{
Switching asthma patients to a once-daily inhaled steroid improves compliance and reduces healthcare costs
}

\author{
Julian F. Guest*, Alison M. Davie, Francis J. Ruiz, Mark J. Greener
}

Catalyst Health Economics Consultants, 34b High Street, Northwood, Middlesex HA6 1BN, UK

Received 15 June 2004; accepted 7 January 2005

\section{KEYWORDS \\ Asthma; \\ Compliance; \\ Costs; \\ Economics; \\ Inhaled steroids; \\ Resource uie}

\begin{abstract}
Objective: To determine the costs and consequenles of sitching asthma patients, managed in primary care, from a twi-tdarly innaled corticosteroid (ICS), to either a once-daily or another givice- darity ICS.

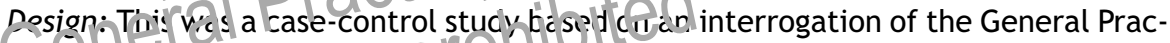
tic= Research Database in the $\mathrm{UK}_{\mathrm{K}}$. for patients with a Read code of asthma who were manage $d \mathrm{~b} b \mathrm{tw} \in \mathrm{E} n \mathrm{1} 990$ and 2001 , and who had received at least two prescript.c nj for a twice-daily ICS within 12 months, before switching to a once-daily ICS (cases) or another twice-daily ICS (controls). Data on resource use was collected for one year before and after the switch. Patients were stratified according to whether their treatment step had been stepped up, stepped down or remained unchanged. Setting: A modelling study performed from the perspective of the UK's National Health Service (NHS).

Main outcome measures: Compliance with ICS, and the cost of drug and non-drug resource use, for the year before and after the switch.

Results: Switching patients managed in primary care to a once-daily ICS increased compliance and reduced NHS costs, irrespective of whether patients' treatment had been stepped up or down. Switching patients to another twice-daily ICS increased compliance to a lesser extent, and increased NHS costs. We believe that this paper offers the first documented association between compliance in asthma and NHS management costs.

Conclusions: Compliance and management costs among patients with asthma managed in primary care appear to be related to both changing treatment and dosing regimen. Within the limitations of our study, the results suggest that patients who are switched to a once-daily ICS rather than another twice-daily preparation are better compliers with their ICS medication. Additionally, patients who become high-
\end{abstract}

\footnotetext{
* Corresponding author. Tel.: +44 1923 450045; fax: +44 1923450046.

E-mail address: julian.guest@catalyst-health.co.uk (J.F. Guest).
} 
compliers after being switched to a once-daily ICS incur lower management costs than patients who become high-compliers after being switched to another twice-daily ICS. These findings should now be investigated further under more controlled conditions. (c) 2005 General Practice Airways Group. Published by Elsevier Ltd. All rights reserved.

\section{Introduction}

Improving compliance with anti-asthma medication is central to improving clinical and economic outcomes for the $5 \%$ of people aged between 20 and 44 years and the $10 \%$ of children who suffer from the disease [1]. However, it has been estimated previously that only $40 \%$ of asthma patients are compliant with their medication over five weeks; half the patients in one study underused their medication and $10 \%$ overused their inhaler [2]. In another study, compliance declined from $51 \%$ during the first week of treatment to less than $30 \%$ after 10 weeks [3]. Non-compliance does not always lead to disease exacerbation. Nevertheless, enhancing compliance is clearly central to improving clinical and economic outcomes in general practice.

Treatment complexity is considered one of the best predictors of compliance [4]. When introduced in the mid-1970s, patients used ICS treatment threc or four times daily. During the 1980s mostriate ents followed a twice-daily reginoth [5]. Coday, increas

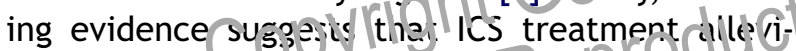
ates symptons and enhances $\mathrm{q}$ AlEv of ire when given once-daily, especially in patients with mildto-moderate asthma [6]. We have previously reported that compliance improves when patients switch from a twice-daily to a once-daily ICS [7].

These studies are part of a growing and compelling body of evidence suggesting that reducing the number of daily doses of ICS frequently improves symptom control, adherence, quality of life, patient satisfaction and costs, across several disease areas [8]. In an analysis of 76 studies that used electronic monitoring, compliance was $79 \%$ with once-daily dosing regimens, compared to $65 \%$ and $51 \%$ with three- and four-times daily dosing, respectively [9].

Asthma accounts for between 1-2\% of total healthcare expenditure across industrialised countries [10]. Exacerbations of the illness associated with non-compliance can result in hospital admissions and bed occupancy $[11,12]$. Despite this heavy toll, to the best of our knowledge no previous study has documented an association between improved compliance in asthma, and costs of management, from the perspective of the UK's NHS.

Against this background, we compared the costs and consequences of switching asthma patients managed in primary care from a twice-daily inhaled corticosteroid (ICS) to either a once-daily or another twice-daily ICS from the perspective of the UK's NHS. Patients were stratified according to whether their GP or nurse moved their drug treatment step up or down, or whether it remained unchanged after the switch.

\section{Methods}

\section{Study design}

This was a retrospective, case-control design study of asthma patients $(n=222)$ who were managed in primary care between 1990 and 2001, who had received at least two prescriptions ior a twice-daily ICS within the 12 month Defore switching to a oncedaily ICD(cises; $h=104)$ or another twice-daily ICS acontrols; $n=118$ )

\section{Resource utilisation}

The study used compliance rates and quantitative healthcare resource-utilisation estimates obtained from the General Practice Research Database (GPRD), which contains more than 30 million patient years of data [13]. GPRD is the world's largest computerised database of anonymised longitudinal clinical records from over 300 general practices. All relevant Read and OXMIS codes and products were used for the search, and included, but were not limited to, H33xxx, 493xxx, 663xxx and 90Jxxx for asthma attacks and monitoring, and $879 x x x$ for assessing patients' treatment step.

Patients' use of the following resources was estimated from the database: drug prescriptions; GP surgery visits; GP-initiated tests; GP domiciliary visits; outpatient visits; and hospital admissions. Resource use was collected for one year before and af ter the switch. Systematic assumptions were made with respect to poor coding of hospital utilisation pertaining to asthma treatment. Events and prescriptions recorded around the time of hospital admission were used as a marker for hospital resource use.

Cases and controls were matched according to: age at switch; gender; location of general practice; 
compliance level; treatment step; co-morbidities (i.e. the incidence of bronchitis, chronic obstructive pulmonary disease (COPD) and respiratory infections); and duration of asthma medication.

\section{Data analysis}

Patients were stratified according to whether their drug regimen had been stepped up, stepped down, or remained unchanged after the switch. Treatment step was assessed by analysing a patient's asthmarelated medication in the year before and after the switch, based on dose, regimen, number of drugs and corresponding drug class.

Differences in drug and non-drug resource use between cases and controls were tested for statistical significance using a 'goodness-to-fit' Chi-square test, whereby the mean annual amount of resource use, and number of scrips, were weighted according to the number of patients in each group. Differences in patients' age were tested using a Mann Whitney U-test.

Annual resource use and prescribed medication in the year before and after the switch was costed at 2001/02 prices using unit costs obtained from published sources [14-16], from the perspective of the UK's National Health Service (NHS).

\section{Complianco}

ICS compliance was assessed for the year before and after switching by comparing the theoretical and actual duration of the prescription. This was achieved by using the time between prescription issues for inhaled corticosteroids as a proxy for compliance, taking into account prescription size and dosage instruction as previously described [7]. Patients were stratified into three bands:

- High: ICS compliance between $71 \%$ and $100 \%$.

- Medium: ICS compliance between $31 \%$ and $70 \%$.

- Low: ICS compliance between $0 \%$ and $30 \%$.

\section{Ethics approval}

Approval for this study was obtained from the GPRD's Scientific and Ethical Advisory Group.

\section{Results}

The cases and controls were well matched as shown in Table 1, with no significant differences between the two groups in respect of matching criteria. It is noteworthy that $29 \%$ of cases switched to a differ-
Table 1 Patient demographics.

\begin{tabular}{|c|c|c|}
\hline & Cases & Controls \\
\hline $\begin{array}{l}\text { Total number of } \\
\text { patients }\end{array}$ & 104 & 118 \\
\hline Male (\%) & 50 & 47 \\
\hline Female (\%) & 50 & 53 \\
\hline Age (years) & $30.4 \pm 25.7$ & $27.2 \pm 26.6$ \\
\hline $\begin{array}{l}\text { Time on treatment } \\
\text { before switch } \\
\text { (months) }\end{array}$ & $46 \pm 25.2$ & $39 \pm 27.6$ \\
\hline \multicolumn{3}{|l|}{$\begin{array}{l}\text { Proportion switching to } \\
\text { the same/different } \\
\text { inhaled steroid }\end{array}$} \\
\hline Same (\%) & 71 & 0 \\
\hline Different (\%) & 29 & 100 \\
\hline \multicolumn{3}{|l|}{$\begin{array}{l}\text { Incidence of } \\
\text { co-morbidities (year } \\
\text { before switch) }\end{array}$} \\
\hline Bronchitis & $7.7 \%$ & $8.5 \%$ \\
\hline COPD & $2.9 \%$ & $1.7 \%$ \\
\hline $\begin{array}{l}\text { Respiratory infection } \\
\text { Incidence of } \\
\text { co-morbidities (year } \\
\text { after switch) }\end{array}$ & $31.7 \%$ & $35.6 \%$ \\
\hline Bronchitis & R. $0 \%$ & $5.1 \%$ \\
\hline COPD & $6.8 \%$ & $1.7 \%$ \\
\hline Respiratcivinisetion & $28.8 \%$ & $21.2 \%$ \\
\hline
\end{tabular}

\section{cllo wited}

er $t$ ics while the other $71 \%$ of cases switched from a twice-daily to a once-daily formulation of the same drug.

Respiratory infections were the primary comorbidity for both groups. $32 \%$ of cases and $36 \%$ of controls experienced an infection before the switch, and after the switch this decreased to $29 \%$ and $22 \%$ respectively.

\section{Compliance}

Compliance improved significantly among cases whose drug treatment was stepped up in the year after the switch, with a $67 \%$ increase in the proportion of high-complying patients. Similarly, there was a $55 \%$ increase in the proportion of highcompliant controls in the year after the switch (see Table 2). However, there were no significant differences in compliance between cases and controls in the year after the switch.

There were no significant differences in compliance among cases whose drug treatment remained unchanged in the year after the switch. However, compliance among controls improved significantly, with a $55 \%$ increase in the proportion of mediumcompliers and a $36 \%$ decrease in the proportion of low-compliers in the year after the switch. More- 
Table 2 Compliance distribution stratified by treatment step.

\begin{tabular}{|c|c|c|c|c|}
\hline & \multicolumn{2}{|c|}{$\begin{array}{l}\text { Percentage of patients in a } \\
\text { compliance band in the year } \\
\text { before the switch }\end{array}$} & \multicolumn{2}{|c|}{$\begin{array}{l}\text { Percentage of patients in a } \\
\text { compliance band in the year } \\
\text { after the switch }\end{array}$} \\
\hline & Cases (\%) & Controls (\%) & Cases (\%) & Controls (\%) \\
\hline \multicolumn{5}{|c|}{ Treatment stepped up } \\
\hline High & $37.1^{*}$ & $37.7^{* *}$ & $62.9^{*}$ & $58.5^{* *}$ \\
\hline Medium & 45.7 & 45.3 & 22.9 & 32.1 \\
\hline Low & 17.1 & 17.0 & 14.3 & 9.4 \\
\hline \multicolumn{5}{|c|}{ Treatment unchanged } \\
\hline High & 63.3 & 61.1 & $70.0^{\text {sut* }}$ & $55.6^{\text {*at }}$ \\
\hline Medium & 20.0 & $22.2^{*+* k+1}$ & 23.3 & $38.9^{*+* * *}$ \\
\hline Low & 16.7 & $16.7^{+3+1+a t}$ & 6.7 & $5.6^{\text {tatson}}$ \\
\hline \multicolumn{5}{|c|}{ Treatment stepped down } \\
\hline High & 61.5 & 61.7 & 69.2 & 66.0 \\
\hline Medium & 25.6 & 25.5 & 20.5 & 25.5 \\
\hline Low & 12.8 & 12.8 & 10.3 & 8.5 \\
\hline $\begin{array}{rl}* & p<0.00 \\
* * * & p<0.05 \\
*^{* * *} p & <0.05 \\
* * * * & p<0.00 \\
*^{* * * *} p & <0.00\end{array}$ & & & & \\
\hline
\end{tabular}

over, there were significantly more high-compliers among cases than controls in the year after/the switch (Table 2).

There were no significant differences among pa tients whose drig treatment wass steppe(d) cover in the year after the switch.

\section{Healthcare resource use and corresponding costs}

Patients whose drug treatment was stepped up Resource use among patients whose drug treatment was stepped up is shown in Table 3 . There were minimal differences between cases and controls before and after the switch in any of the compliance bands. Most notably, non-drug healthcare resource use did not differ between cases and controls, in corresponding compliance bands, in the years before or after the switch. However, highcompliant cases $(p<0.001)$ and controls $(p<0.01)$ made more GP visits than patients in the mediumand low-compliance bands in the year before the switch. Additionally, after the switch, cases in the high-compliance band received fewer $(p<0.005)$, and low-compliers received more $(p<0.01)$, prescriptions for inhaled steroids than controls. All other prescribing patterns were comparable (Fig. 1).

The total cost of managing high-compliant cases decreased by $14 \%$ in the year after switching (from
$£ 690$ to 1595$)$, I Fenlecting a 31\% decline in non-drug D-escurce costs and ai $11 \%$ increase in drug costs. The total cost on managing controls increased by $67 \%$ arter switching (from $£ 480$ to $£ 801$ ), due to increases of $161 \%$ and $14 \%$ in medication and in nondrug resource costs, respectively (Fig. 2).

The total annual cost of managing mediumcompliant cases and controls increased by $30 \%$ (from $£ 382$ to $£ 495$ ) and $14 \%$ (from $£ 301$ to $£ 342$ ) respectively, after the switch. This was due to increases in drug costs of $85 \%$ (cases) and $27 \%$ (controls), as well as higher costs of non-drug resource use by $8 \%$ (cases) and $1 \%$ (controls).

The total annual cost of managing low-compliers increased by $170 \%$ (from $£ 154$ to $£ 415$ ) among cases and decreased by $30 \%$ (from £175 to £125) among controls after the switch. This was due to a $179 \%$ and $159 \%$ increase in drug and non-drug resource costs, respectively, among cases in the year after the switch, compared to a $24 \%$ increase in drug costs and a $47 \%$ decrease in non-drug resource costs among controls.

\section{Patients whose drug treatment step remained unchanged}

Resource use among patients whose drug treatment remained unchanged is shown in Table 3 . There were minimal differences between cases and controls before and after the switch in any of the compliance bands. Most notably, non-drug health- 
Table 3 Mean resource use, stratified by compliance and treatment step.

\begin{tabular}{|c|c|c|c|c|c|c|c|c|c|}
\hline & \multicolumn{9}{|c|}{ Resource use per patient } \\
\hline & \multicolumn{3}{|c|}{ High compliance band } & \multicolumn{3}{|c|}{ Medium compliance band } & \multicolumn{3}{|c|}{ Low compliance band } \\
\hline & Case & Control & $p$ & Case & Control & $p$ & Case & Control & $p$ \\
\hline \multicolumn{10}{|c|}{ Patients whose drug treatment was stepped up } \\
\hline Before switch & $(n=13)$ & $(n=20)$ & & $(n=16)$ & $(n=24)$ & & $(n=6)$ & $(n=9)$ & \\
\hline $\mathrm{GP}$ visits & 14.69 & 10.65 & ns & 7.56 & 8.00 & ns & 4.17 & 6.22 & ns \\
\hline Hospital admissions & 0.08 & 0.00 & $\mathrm{n} / \mathrm{a}$ & 0.00 & 0.00 & $\mathrm{n} / \mathrm{a}$ & 0.00 & 0.00 & $\mathrm{n} / \mathrm{a}$ \\
\hline Outpatient visits & 0.85 & 0.50 & ns & 1.13 & 0.75 & ns & 1.33 & 1.22 & ns \\
\hline GP-initiated tests & 0.00 & 0.00 & $\mathrm{n} / \mathrm{a}$ & 0.13 & 0.46 & $\mathrm{n} / \mathrm{a}$ & 0.00 & 0.33 & $\mathrm{n} / \mathrm{a}$ \\
\hline GP domiciliary visits & 0.08 & 0.00 & $\mathrm{n} / \mathrm{a}$ & 0.00 & 0.08 & $\mathrm{n} / \mathrm{a}$ & 0.00 & 0.22 & $\mathrm{n} / \mathrm{a}$ \\
\hline After switch & $(n=22)$ & $(n=31)$ & & $(n=8)$ & $(n=17)$ & & $(n=5)$ & $(n=5)$ & \\
\hline GP visits & 10.95 & 13.81 & ns & 8.75 & 8.35 & ns & 10.80 & 3.80 & ns \\
\hline Hospital admissions & 0.05 & 0.03 & $\mathrm{n} / \mathrm{a}$ & 0.00 & 0.00 & $\mathrm{n} / \mathrm{a}$ & 0.00 & 0.00 & $\mathrm{n} / \mathrm{a}$ \\
\hline Outpatient visits & 1.18 & 1.52 & ns & 0.25 & 0.41 & ns & 0.40 & 0.80 & ns \\
\hline GP-initiated tests & 0.18 & 0.29 & $\mathrm{n} / \mathrm{a}$ & 0.13 & 0.41 & $\mathrm{n} / \mathrm{a}$ & 0.00 & 0.00 & $\mathrm{n} / \mathrm{a}$ \\
\hline GP domiciliary visits & 0.00 & 0.00 & $\mathrm{n} / \mathrm{a}$ & 0.00 & 0.00 & $\mathrm{n} / \mathrm{a}$ & 0.00 & 0.00 & $\mathrm{n} / \mathrm{a}$ \\
\hline \multicolumn{10}{|c|}{ Patients whose treatment step remained unchanged } \\
\hline Before switch & $(n=19)$ & $(n=11)$ & & $(n=6)$ & $(n=4)$ & & $(n=5)$ & $(n=3)$ & \\
\hline GP visits & 8.68 & 10.82 & ns & 7.50 & 12.50 & ns & 4.60 & 14.67 & ns \\
\hline Hospital admissions & 0.05 & 0.05 & $\mathrm{n} / \mathrm{a}$ & 0.00 & 0.00 & $\mathrm{n} / \mathrm{a}$ & 0.20 & 0.00 & $\mathrm{n} / \mathrm{a}$ \\
\hline Outpatient visits & 0.89 & 0.91 & ns & 0.17 & 0.00 & ns & 1.60 & 0.11 & ns \\
\hline GP-initiated tests & 0.21 & 0.09 & $\mathrm{n} / \mathrm{a}$ & 0.00 & 2.00 & $\mathrm{n} / \mathrm{a}$ & c.nc & 0.67 & $\mathrm{n} / \mathrm{a}$ \\
\hline GP domiciliary visits & 0.00 & 0.00 & $\mathrm{n} / \mathrm{a}$ & 0.00 & 0.00 & & 0.00 & 0.33 & $\mathrm{n} / \mathrm{a}$ \\
\hline After switch & $(n=21)$ & $(n=10)$ & & $(n=7)$ & 4 & & $(n=2)$ & $(n=1)$ & \\
\hline GP visits & 8.48 & 12.40 & & 11 & 13.29 & & 4.00 & 1.00 & ns \\
\hline Hospital admissions & 0,05 & 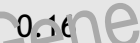 & r.ta & 0.00 & 0.29 & r.á & 0.00 & 0.00 & $\mathrm{n} / \mathrm{a}$ \\
\hline Outpatient visits & c.5:L & 0.40 & rs & (1) $x$ & 1.57 & ns & 0.00 & 0.00 & ns \\
\hline GP-initia ted $\mathrm{t}=\mathrm{st} \mathrm{s}$ & 0.00 & 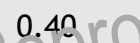 & $n / a$ & 0.43 & 1.57 & $\mathrm{n} / \mathrm{a}$ & 0.00 & 0.00 & $\mathrm{n} / \mathrm{a}$ \\
\hline GP domiciitiary visits & 0.00 & & $\mathrm{n} / \mathrm{a}$ & 0.00 & 0.00 & $\mathrm{n} / \mathrm{a}$ & 0.00 & 0.00 & $\mathrm{n} / \mathrm{a}$ \\
\hline \multicolumn{10}{|c|}{ Patients whose drug treatment was stepped down } \\
\hline Before switch & $(n=24)$ & $(n=29)$ & & $(n=10)$ & $(n=12)$ & & $(n=5)$ & $(n=6)$ & \\
\hline GP visits & 13.25 & 12.28 & ns & 6.20 & 11.92 & ns & 5.20 & 10.33 & ns \\
\hline Hospital admissions & 0.04 & 0.00 & $\mathrm{n} / \mathrm{a}$ & 0.00 & 0.00 & $\mathrm{n} / \mathrm{a}$ & 0.00 & 0.00 & $\mathrm{n} / \mathrm{a}$ \\
\hline Outpatient visits & 1.13 & 0.55 & ns & 0.10 & 0.75 & $<0.01$ & 0.20 & 1.17 & ns \\
\hline GP-initiated tests & 0.17 & 0.07 & $\mathrm{n} / \mathrm{a}$ & 0.10 & 1.00 & $\mathrm{n} / \mathrm{a}$ & 0.00 & 1.83 & $\mathrm{n} / \mathrm{a}$ \\
\hline GP domiciliary visits & 0.04 & 0.07 & $\mathrm{n} / \mathrm{a}$ & 0.00 & 0.00 & $\mathrm{n} / \mathrm{a}$ & 0.00 & 0.67 & $\mathrm{n} / \mathrm{a}$ \\
\hline After switch & $(n=27)$ & $(n=31)$ & & $(n=8)$ & $(n=12)$ & & $(n=4)$ & $(n=4)$ & \\
\hline GP visits & 10.44 & 12.94 & ns & 7.00 & 7.92 & ns & 3.00 & 3.50 & ns \\
\hline Hospital admissions & 0.07 & 0.00 & $\mathrm{n} / \mathrm{a}$ & 0.00 & 0.25 & $\mathrm{n} / \mathrm{a}$ & 0.00 & 0.00 & $n / a$ \\
\hline Outpatient visits & 0.93 & 0.42 & ns & 0.63 & 0.92 & ns & 0.00 & 1.00 & ns \\
\hline GP-initiated tests & 0.11 & 0.13 & $\mathrm{n} / \mathrm{a}$ & 0.13 & 0.00 & $\mathrm{n} / \mathrm{a}$ & 0.00 & 0.25 & $n / a$ \\
\hline GP domiciliary visits & 0.00 & 0.19 & $\mathrm{n} / \mathrm{a}$ & 0.00 & 0.08 & $\mathrm{n} / \mathrm{a}$ & 0.00 & 0.00 & $\mathrm{n} / \mathrm{a}$ \\
\hline
\end{tabular}

care resource use did not differ between cases and controls in corresponding compliance bands in the years before or after the switch, and there was no significant change within the groups as a result of the switch. However, after the switch, highcompliant cases received fewer prescriptions for oral steroids $(p<0.05)$ and xanthines $(p<0.005)$ than controls, although use of the former declined among controls $(p<0.05)$. All other prescribing patterns were comparable (Fig. 3).
The total cost of managing high-compliant cases decreased by $6 \%$ in the year after switching (from $£ 563$ to £528), due to decreases of $13 \%$ and $2 \%$ in drug and non-drug resource costs, respectively. However, the total cost of managing controls increased by $40 \%$ after switching (from $£ 473$ to $£ 654$ ), due to a $55 \%$ increase in medication costs and a $27 \%$ increase in non-drug resource costs (Fig. 4).

Furthermore, the total cost of managing medium-compliant cases decreased by $2 \%$ after the 


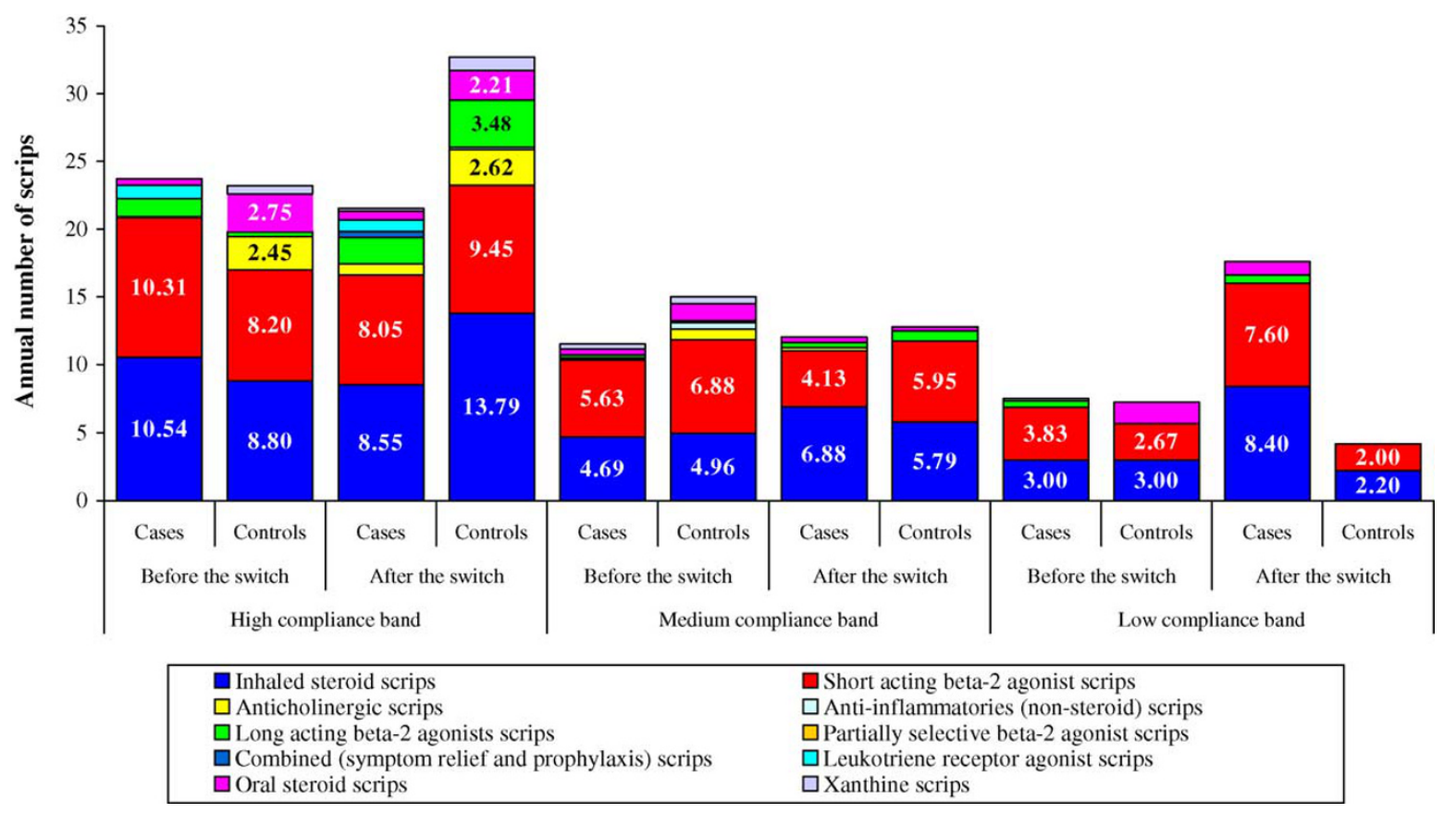

Figure 1 Annual number of prescriptions for patients whose drug treatment was stepped up.

switch (from $£ 521$ to £511). Non-drug costs de- year after the sivigh/ (Trom $£ 262$ to $£ 457$ ), due clined by $49 \%$ but drug costs rose by $263 \%$. The to 230 decrease in non-drug resource costs and cost of managing medium-compliant controls if $2.449 \%$ increase in arug costs. This compared to

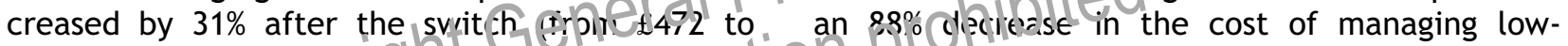
$£ 618$ ) due to an $8 \%$ decrease in urug costs land a 1 (cmpiant controls (from £343 to £41) due to de$64 \%$ increase in nor-crug costs The CDSOffrinanaging low-compliant cases increased by $74 \%$ in the creases of $54 \%$ and $94 \%$ in drug and non-drug costs, respectively.

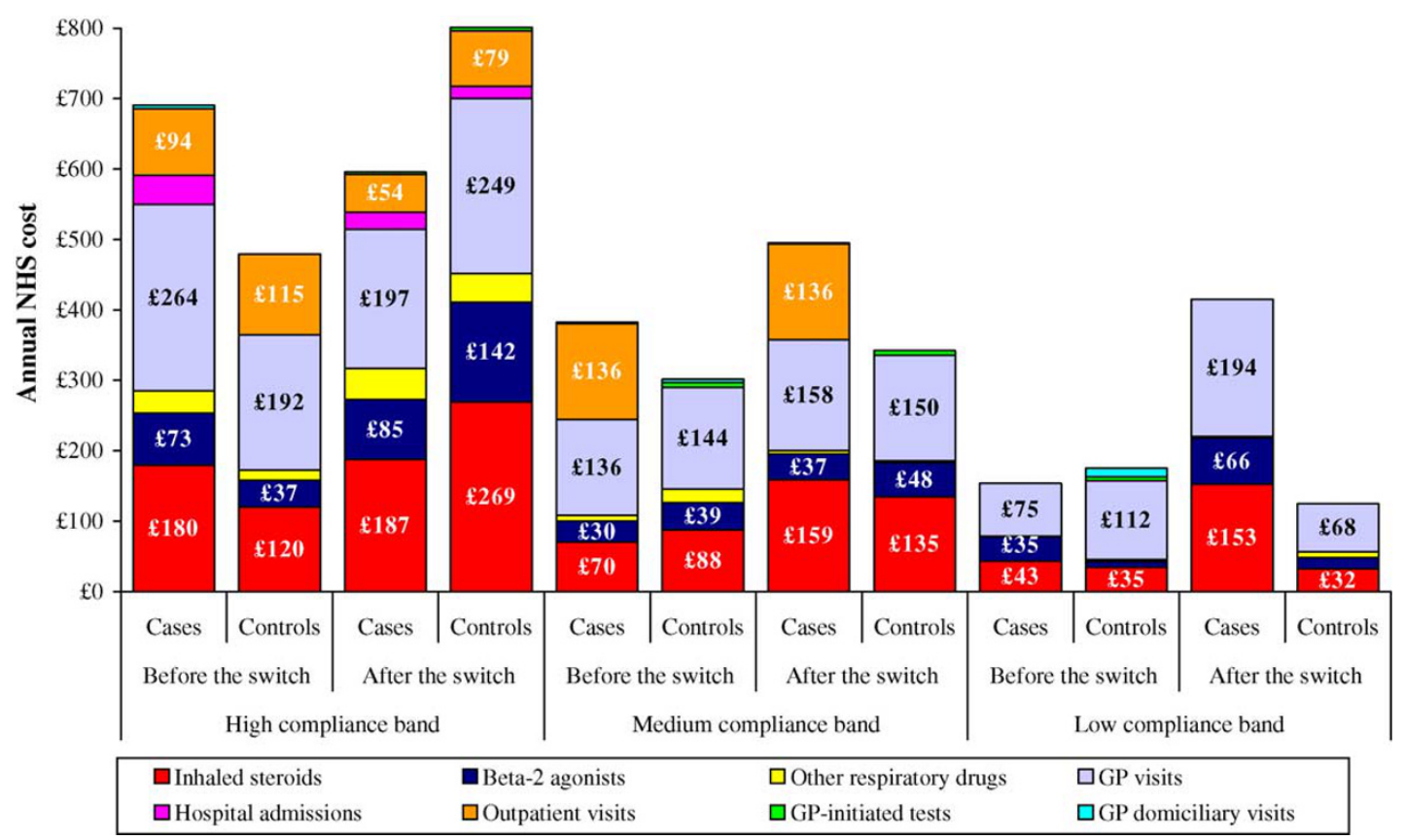

Figure 2 Mean NHS cost of resource use for patients whose drug treatment was stepped up. 


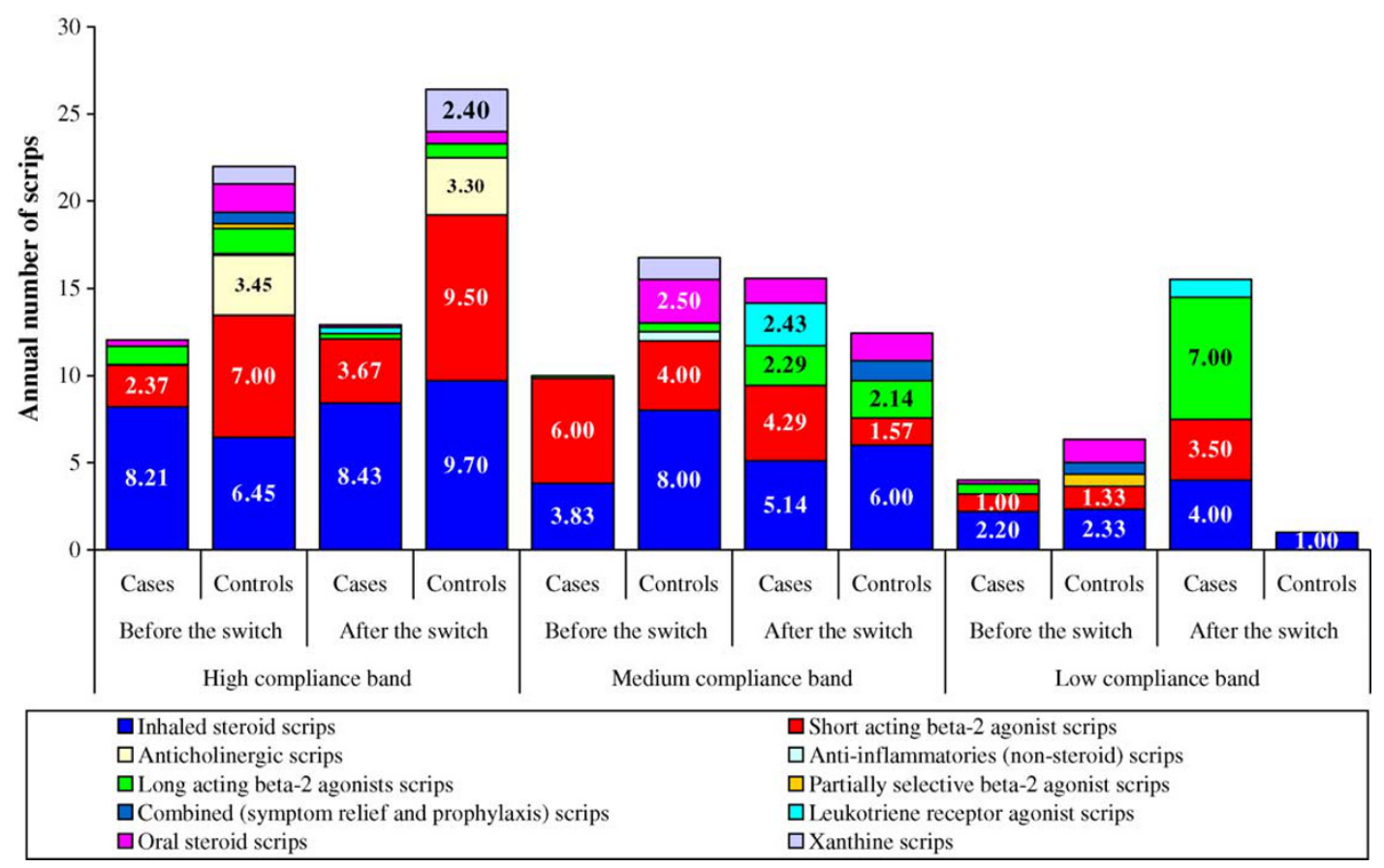

Figure 3 Annual number of prescriptions for patients whose drug treatment step remained unchanged.

\section{Patients whose drug treatment was stepped} down

Resource use among patients whose drug treatmen was stepped down is shov'n in Tabto 3, The were minimal differences $k \in t w \in e r$ cases and controls be. 0 fore and aftertne switch in a fiv of ci) Compriance bands. Mast poptasy, non-úug healthcare resource disecidid roldifer between cases and controls in corresponding formpliance bands in the years before oir atter the switch (except for the number of outpatient visits among medium-compliers in the year before the switch), and there was no sig-

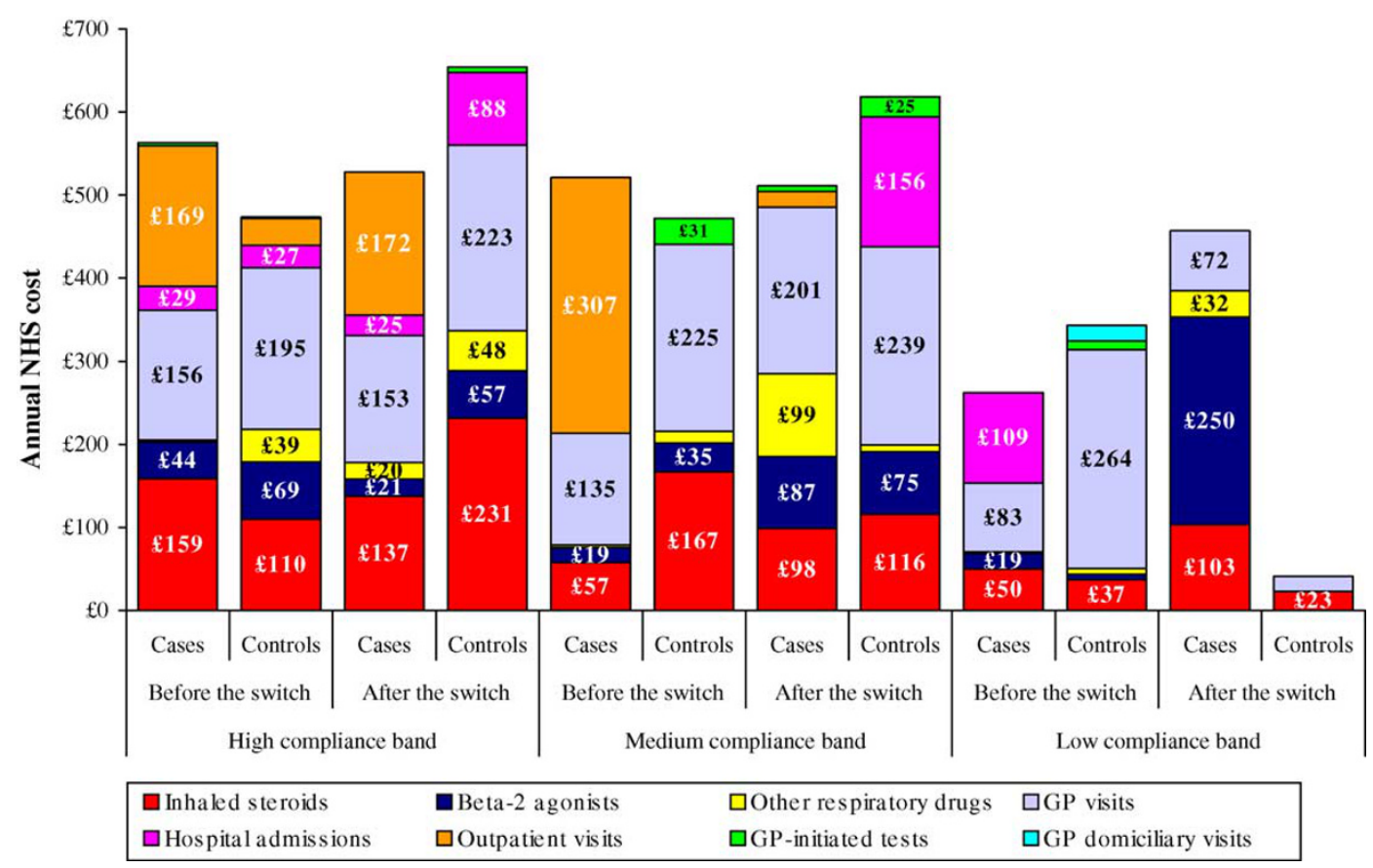

Figure 4 Mean NHS cost of resource use for patients whose drug treatment step was unchanged. 


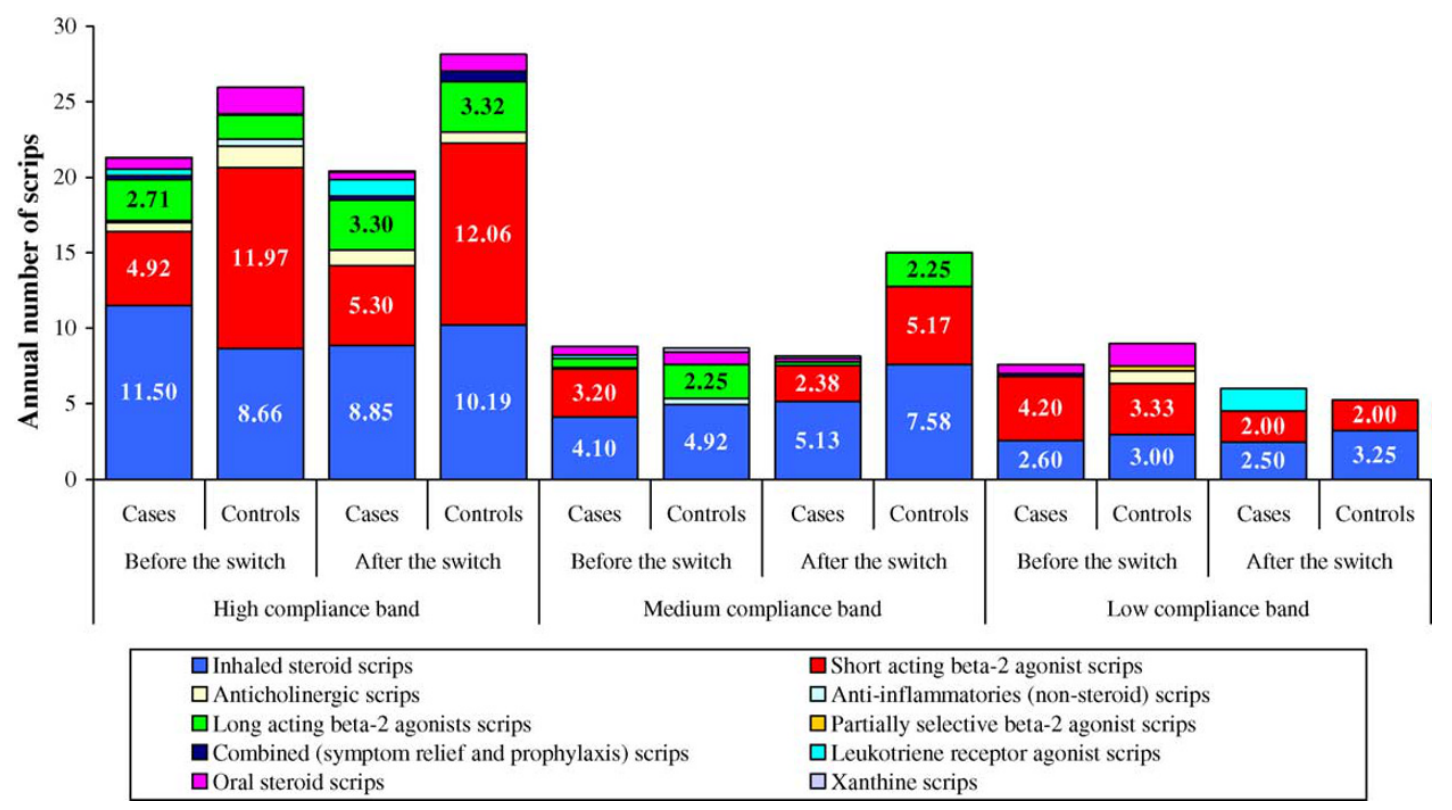

Figure 5 Annual number of prescriptions for patients whose drug treatment was stepped down.

nificant change within the groups as a result of the switch. However, high-compliant cases received fewer $(p<0.01)$ prescriptions for short-acting beta2 agonists in both years compared to controls. High-compliant cases also received more $(p-\theta)$ (1) leukotriene prescriptions than conton in the year after the switch Nedilin-compliant cases reseived fewer $(p<0.05)$ in naled steroic restipipions during the year after the switch than controls. All other prescribing patterns were comparable (Fig. 5).

The total cost of managing high-compliant cases decreased by $23 \%$ in the year after switching (from $£ 774$ to $£ 597$ ), due to a reduction of $17 \%$ and $27 \%$ in drug and non-drug resource costs, respectively. The total cost of managing controls increased by $41 \%$ after the switch (from $£ 509$ to $£ 717$ ), due to increases of $38 \%$ and $44 \%$ in drug and non-drug resource costs (Fig. 6).

The total annual cost of managing mediumcompliant cases and controls increased by $31 \%$ (from $£ 292$ to $£ 384$ ) and 19\% (from $£ 423$ to £479) respectively, after the switch. This was due to increases in drug costs of $10 \%$ (cases) and 13\% (controls) and non-drug resource use costs of $41 \%$ and $23 \%$, respectively. Furthermore, the total annual cost of managing low-compliant cases increased by $24 \%$ after the switch (from £159 to £197), due to a $119 \%$ increase in drug costs and a $42 \%$ decrease in non-drug resource costs. The annual cost of managing comparable controls decreased by $52 \%$ (from $£ 288$ to £139), due to a $73 \%$ decrease in non-drug resource costs and a $102 \%$ increase in drug costs (Fig. 6).

\section{Discussion}

The costs of healticale have assumed increasing innditance in recent years, and this has been reflected in the $d=$ nind for studies that are cognisant of the likely economic impact of an intervention, as well as its potential clinical benefits. By stratifying patients according to their treatment step, our analysis found that proportionally more patients become high-compliers for less NHS cost when their treatment is stepped up or down, by switching to a once-daily inhaled steroid rather than another twice-daily preparation.

We previously reported that compliance was significantly improved when patients switch from taking a twice-daily to a once-daily inhaled steroid [7]. Additionally, once-daily inhaled steroids have been shown to improve compliance due to the easy, burden-free dosing regimen [17]. Patients have solely to decide at what point during the day they should administer their medication. Research suggests afternoons or evenings to be most effective [5]. Apart from these studies, our literature review found very few other studies assessing the economic impact of switching from a twice-daily to a once-daily inhaled steroid.

\section{Patients whose drug treatment was stepped up}

In this study, compliance was found to be comparable between cases and controls whose treatment 


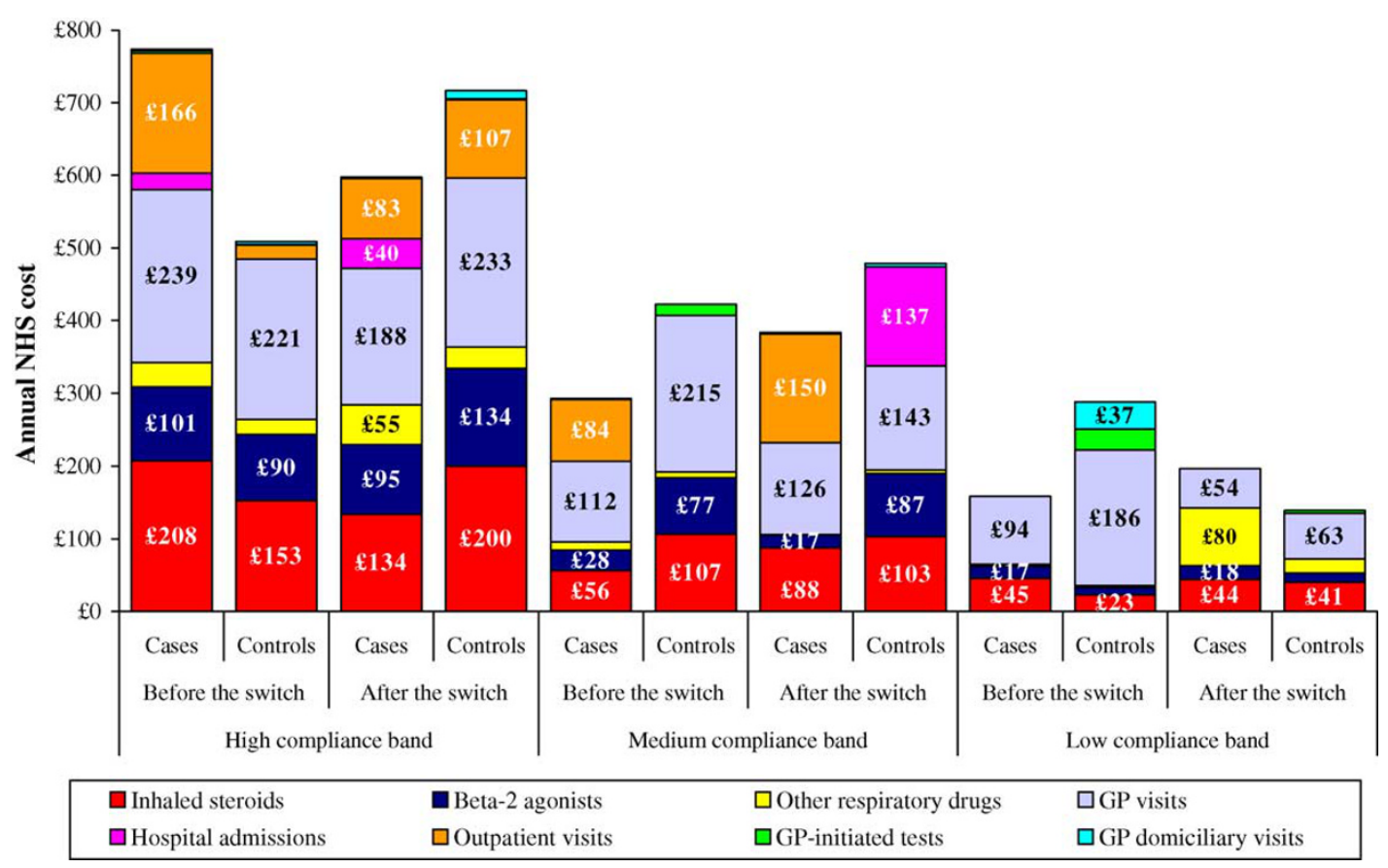

Figure 6 Mean NHS cost of resource use for patients whose drug treatment was stepped up.

was stepped up in the year after the switch. Moving up a step may underscore to a patient that their asthma was poorly controlled. The number of IrS prescriptions decreased for high-complian cases in the year after the switkf, esuting in a significant difference (ised (ig, 1) between the nurnoer of ICS preseripuions for cases Arie Controts in the high-compliance band. Eviderice suggests that increasing medication improves patients' symptoms and quality of life, and that poor inhaler technique resulting in a lower respirable dose of ICS can be managed efficiently by stepping patients up [4]. Hence, both could enhance compliance. The reduction in ICS prescriptions for high-compliant cases could suggest that stepping asthma patients up in their treatment has improved their symptoms and quality of life.

The study further illustrates that when treatment is stepped up, the cost of managing highcompliant cases after being switched to a oncedaily ICS is less than that of managing highcompliant controls after they have been switched to another twice-daily ICS. Cases in the highcompliance band made fewer GP visits and received less ICS prescriptions in the year after the switch, resulting in a $14 \%$ decrease in the cost of managing these patients. However, comparable controls made more GP visits and received more ICS prescriptions, thus increasing management costs by $67 \%$. A reduction in costs associated with simpler dosing regimens has been reported in other studies [8].

\section{Patients whose drus tien finerit step} remained unchased

Proportioniäliy thine cases whose treatment step $r \in$ arined unchanged in the year after the switch became high-compliers compared to controls. However, proportionally more controls became medium-compliers in the year after the switch. These changes in compliance were associated with a decrease in the cost of managing high-compliant cases, and an increase in the costs of managing high- and medium-compliant controls. This suggests that compliance improves if treatment regimens are simplified, a finding consistent with several other studies $[6,9,18,19]$. Patients continuing with a twice-daily dosing regimen may have less motivation and willingness to comply with treatment than those following a once-daily regimen [12].

\section{Patients whose drug treatment was stepped down}

Compliance among cases and controls was found to be comparable in the year after the switch for those whose treatment was stepped down. Highcompliant cases received fewer ICS prescriptions in the year after the switch, and fewer than their matched controls. They also received fewer prescriptions for short-acting beta-2 agonists in the year after the switch, but more prescriptions for leukotrienes. Furthermore, these changes in pre- 
scription quantities for the latter two preparations were significantly different to the prescription changes for high-compliant controls (see Fig. 5). Additionally, high-compliant cases made fewer GP visits, but more outpatient visits, in the year after the switch compared to high-compliant controls whose treatment was stepped down.

The differences between high-compliant cases and controls when treatment is stepped down showed in the costs of managing these patients in the year after the switch. Cases in the highcompliance band incurred a decrease in management costs $(23 \%)$, as compared to high-compliant controls whose management costs increased by $31 \%$. This resulted in the reduced costs of managing high-compliant cases after switching to a oncedaily ICS, as compared to the cost of managing highcompliant controls after they had been switched to another twice-daily ICS.

These findings add to existing evidence suggesting that the aim of asthma management is to increase and maintain a high level of compliance, in order to achieve maximum asthma control with the lowest possible dose of ICS.

\section{Study limitations}

This study employed a caselopht foi tesign in order to make comparions between switching-patierts from a twice-dariy innaled ster id (t) a lonce-daily regimen or to another twice-daily inhaled steroid, in terms of resource use and compliance with ICS. This design resulted in small numbers of patients eligible for analysis. Nevertheless, we believe this paper offers the first documented association between improved compliance in asthma and NHS management costs. Notwithstanding this, our study is subject to a number of limitations.

This was a retrospective, observational study and GPs may have had a multiplicity of reasons for changing treatment. Patients were not randomised to treatment, and patients may have had numerous reasons for altered behaviour which may relate only marginally to altered treatment. The study relies on surrogate markers of compliance, rather than direct measures. Moreover, the analysis does not disentangle other factors employed by physicians in general practice that might influence compliance, such as clinical feedback [3] or motivational models [20]. Neither does the study consider the impact of different devices available for delivering the ICS dose. The analysis was unable to consider under- or over recording of asthma-related resource use, and systematic assumptions were made with respect to asthma resource utilisation in the presence of poor coding and the absence of clinical expertise. Additionally, the potential for some patients in the data set to have COPD, misdiagnosed as asthma, cannot be eliminated. GPs and nurses are still less confident about diagnosing COPD as compared to asthma [21]; hence, informed decisions with regard to misdiagnosis could not be made.

Cases and controls were matched according to age, gender, location of general practice, compliance level, treatment step, co-morbidities, and duration of asthma medication, at the time of switching. However, patients were not matched according to baseline use of healthcare resources, as this potentially could have reduced the already small numbers. Nevertheless, our analysis found that there were no significant differences in resource use between cases and controls in the year before the switch, except for outpatient visits among mediumcompliers whose treatment was stepped down after the switch.

Despite these limitations, the data contained within the GPRD, whilst retrospective, reflect clinical practice on a large number of patients without the selection biases assnciated $v$ ith inclusion criteria that may arise jroin envention studies [13]. Hence, this study (rep:esents patients' management innaturaliscic general practice, rather than an artiticial clinical torial (1) Which patients may alter their fompliande by being aware that they could be assessed. As a result, we believe our findings support the notion that compliance and management costs are related to both changing treatment and dosing regimen. These findings should now be investigated further under more controlled conditions.

\section{Conclusion}

Compliance and management costs among patients with asthma managed in primary care appear to be related to both changing treatment and dosing regimen. Within the limitations of our study, the results suggest that patients who are switched to a oncedaily ICS rather than another twice-daily preparation are better compliers with their ICS medication. Additionally, patients who become high-compliers after being switched to a once-daily ICS incur lower management costs than patients who become highcompliers after being switched to another twicedaily ICS. We hope that our findings can help reduce the clinical and economic toll arising from poorly controlled, poorly-compliant asthma patients.

\section{Conflict of interest statement}

This research was funded by Schering-Plough; however, they had no input into the analysis of the 
results or manuscript. The authors have no conflicts of interest that are directly relevant to the content of this manuscript.

\section{References}

[1] Tattersfield AE, Knox AJ, Britton JR, et al. Asthma. Lancet 2002;360:1313-22.

[2] Chmelik F, Doughty A. Objective measurements of compliance in asthma treatment. Ann Allergy 1994;73:52732.

[3] Onyirimba F, Apter A, Reisine S, et al. Direct clinician-topatient feedback discussion of inhaled steroid use: its effect on adherence. Ann Allerg Asthma Immunol 2003;90:4115.

[4] Hyland ME. Types of non-compliance. Eur Respir Rev 1998;8:255-9.

[5] Self TH, Sameri RM. Efficacy of inhaled corticosteroids administered once daily for asthma. Amer Journ Mang Care 2003;9:91-102.

[6] Casale TB, Nelson HS, Kemp J, et al. Budesonide turbuhaler delivered once daily improves health-related quality of life and maintains improvements with a stepped-down dose in adults with mild to moderate asthma. Ann Allerg Asthma Immunol 2003;3:323-30.

[7] Das Gupta R, Guest JF. Factors affecting UK primary-care costs of managing patients with asthma over 5 years. PharmacoEconomics 2003;21:357-69.

[8] Richter A, Anton SE, Koch P, et al. The impact of re ducing dose frequency on health outcomes Elin fine 2003;25:2307-36.

[9] Claxton AJ, Cramer J Pieice C A cystematic review of the associations ot Ween dose regimens and medi at on com pliance. Elin ther 2001;23:1296-21?
[10] Haahtela T. The disease management approach to controlling asthma. Respir Med 2002;96(Suppl A):S1-8.

[11] Bateman ED. Using clinical measures of disease control to reduce the burden of asthma. Pharmacoeconomics 2001;19:7-12.

[12] Bukstein DA, Henk HJ, Luskin AT. A comparison of asthmarelated expenditures for patients started on montelukast versus fluticasone propionate as monotherapy. Clin Ther 2001;23:1589-600.

[13] Wood L, Coulson R. Revitalizing the General Practice Research Database: plans, challenges, and opportunities. Pharmacoepidemiol Drug Safety 2001;10:379-83.

[14] Department of Health. NHS reference costs 2000/2001. Available on-line at http://www.doh.gov.uk/nhsexec/ refcosts.

[15] Netten A, Rees T, Harrison G. Unit costs of health and social care. Canterbury: Personal Social Services Research Unit, University of Kent; 2001.

[16] Monthly Index of Medical Specialities (MIMS). November 2002 edition. Haymarket Medical Ltd, London.

[17] O'Connell EJ. Efficacy of budesonide in moderate to severe asthma. Clin Ther 2002;24(6):887-905, discussion 837.

[18] Boyd G, Abdallah S, Clark R. Twice or four times daily beclomethasone dipropionate in mild stable asthma? Clin Allergy 1985;15:383-9.

[19] Noonan M, Karpel JP, Bensch GW, et al. Comparison of once-daily to twice-daily treatment with mometasone furoate dry powder inhaler. Ann Allergy Asthma Immunol 2001;86:36-43.

[20] Cassidy CA. Using the tran the orecical model to facilitate behaviof chang: In tients with chronic illness. J Am Acad Nurse lract 1999;11:281-7.

[21] Halpin DMG Radoli $M$, on behalf of the BTS COPD Consocitm. Implementing the BTS COPD Guidelines: How far have we come? 2004. Available on-line at http://www.britthoracic.org.uk/copd/pdf/posters_prens/Implementing\% 20the\%20COPD.pdf.

Available online at www.sciencedirect.com

science $\boldsymbol{d}$ Directo

Available online at http://www.thepcrj.com 